\title{
INVARIANT PLURICOMPLEX GREEN FUNCTIONS
}

\author{
MACIEJ KLIMEK \\ Department of Mathematics, Uppsala University \\ P.O. Box 480, S-751 06 Uppsala, Sweden
}

\begin{abstract}
The purpose of this paper is to present a concise survey of the main properties of biholomorphically invariant pluricomplex Green functions and to describe a number of new examples of such functions. A concept of pluricomplex geodesics is also discussed.
\end{abstract}

1. Introduction. Let $\Omega$ be an open bounded subset of $\mathbb{C}$, and let $a$ be a point in $\Omega$. Suppose that a function $z \mapsto G_{\Omega}(z, a)$ has the following properties:

(i) $G_{\Omega}(\cdot, a)$ is non-negative and harmonic in $\Omega \backslash\{a\}$;

(ii) for each $w \in \partial \Omega$ we have $\lim _{z \rightarrow w} G_{\Omega}(z, a)=0$;

(iii) $z \mapsto G_{\Omega}(z, a)+\log |z-a|$ extends to a harmonic function on $\Omega$.

Then the function $z \mapsto G_{\Omega}(z, a)$ is called the classical Green function for $\Omega$ with pole at $a$. If (ii) is replaced by the following condition:

(ii)' there is a polar set $F \subset \partial \Omega$ such that for each $w \in \partial \Omega \backslash F$ we have $\lim _{z \rightarrow w} G_{\Omega}(z, a)=0$,

then $G_{\Omega}(\cdot, a)$ is called the generalized Green function for $\Omega$ with pole at a. Every set $\Omega$ can have at most one Green function with a given pole. Moreover, if $u$ is the solution to the Dirichlet problem

$$
\left\{\begin{array}{l}
u \in \mathcal{C}^{2}(\Omega) \cap \mathcal{C}(\bar{\Omega}) \\
\Delta u=0 \quad \text { in } \Omega, \\
u(z)=\log |z-a| \quad \text { for each } z \in \partial \Omega
\end{array}\right.
$$

then $z \mapsto u(z)-\log |z-a|$ is the classical Green function with pole at $a$. Conversely, if $\Omega$ has the classical Green function, the function $u$ defined by (iii) (and extended continuously to a function on $\bar{\Omega}$ ) solves the Dirichlet problem.

It can be shown that the Green function $G_{\Omega}$ is symmetric, that is $G_{\Omega}(z, w)=$ $G_{\Omega}(w, z)$ if $z \neq w$ and $z, w \in \Omega$. It can also be shown that the functions

1991 Mathematics Subject Classification: 32F05, 32H15.

The paper is in final form and no version of it will be published elsewhere. 
$-G_{\Omega}$ contract holomorphic mappings in the following sense. If $f: \Omega_{1} \longrightarrow \Omega_{2}$ is a holomorphic mapping and both $\Omega_{1}$ and $\Omega_{2}$ have Green's functions, then $\left(-G_{\Omega_{2}}\right)(f(z), f(w)) \leq\left(-G_{\Omega_{1}}\right)(z, w)$. In particular, if $\Omega$ is a bounded simply connected domain, $a \in \Omega$, and $f$ is a conformal mapping which maps $\Omega$ onto the unit disc so that $f(a)=0$, then $G_{\Omega}(z, a)=-\log |f(z)|$ for $z \in \Omega$. Conversely, the Riemann mapping theorem (for a simply connected domain that has a Green function) can be proved by using the Green function.

In $\mathbb{C}^{n}$ one can introduce pluricomplex Green functions which generalize the one-variable Green functions with logarithmic poles. The pluricomplex Green functions in several complex variables display many analogies to the Green functions of the classical potential theory. At the same time, significant differences exist. Probably the most important difference is that pluricomplex Green functions need not be symmetric. A symmetric pluricomplex Green function was introduced by Cegrell [C1, C2]. Its definition will be stated in the next section. Another Green function in several complex variables was introduced by Hervé $[\mathrm{H} 1, \mathrm{H} 2]$. Over the recent years, pluricomplex Green functions have been used in various contexts of complex analysis. The reader is referred to $[\mathrm{A}, \mathrm{A} 2, \mathrm{BL}, \mathrm{B}$, BT3, D2, D, DG, JP1, JP2, K1, K2, K3, KR, L, M, PS, SE] for details.

This paper is a slightly extended version of four lectures given in October 1992 in the International Banach Centre in Warsaw. Although primarily meant as a survey of the main properties of the pluricomplex Green functions, this paper contains also a number of new examples of such functions. In the last section of the paper, we introduce and briefly investigate a concept of pluricomplex geodesics.

Throughout the paper, $\mathbb{D}$ will be used to denote the open unit disc; the symbols $\|\cdot\|$ and $|\cdot|$ will denote the Euclidean norm and the polydisc norm in $\mathbb{C}^{n}$, respectively.

2. Basic properties of pluricomplex Green functions. Let $\Omega$ be a domain in $\mathbb{C}^{n}$, and let $a \in \Omega$. If $u \in \mathcal{P} \mathcal{S H}(\Omega)$, we say that $u$ has a logarithmic pole at $a$ if the function $z \mapsto u(z)-\log |z-a|$ is bounded from above in a neighbourhood of $a$. We shall denote by $\mathcal{G}(\Omega, a)$ the family of all negative plurisubharmonic functions on $\Omega$ which have a logarithmic pole at $a$. Note that if $\Phi: \Omega_{1} \longrightarrow \Omega_{2}$ is a holomorphic mapping and $u \in \mathcal{G}\left(\Omega_{2}, \Phi(a)\right)$, then $u \circ \Phi \in \mathcal{G}\left(\Omega_{1}, a\right)$.

EXAMPLE 2.1. In this example we shall describe $\mathcal{G}(\Omega,(a, b))$ for $\Omega=\{(z, w) \in$ $\left.\mathbb{C}^{2}:|z w|<1\right\}$ and $(a, b) \in \Omega$.

Let $\Omega_{*}=\{(z, w) \in \Omega: z \neq 0\}$ and let $\mathbb{C}_{*}=\mathbb{C} \backslash\{0\}$. Then $(z, w) \mapsto(z, w / z)$ is a biholomorphic mapping from $\mathbb{C}_{*} \times \mathbb{D}$ onto $\Omega_{*}$. Note that

$$
u \in \mathcal{G}\left(\mathbb{C}_{*} \times \mathbb{D},(a, b)\right) \Leftrightarrow \exists v \in \mathcal{G}(\mathbb{D}, b) \forall w \in \mathbb{D}: u(z, w)=v(w) .
$$

Consequently,

$$
u \in \mathcal{G}\left(\Omega_{*},(a, b)\right) \Leftrightarrow \exists v \in \mathcal{G}(\mathbb{D}, a b) \forall w \in \mathbb{D}: u(z, w)=v(z w) .
$$


Since closed pluripolar sets are removable singularities for bounded plurisubharmonic functions and $\Omega$ is symmetric, we conclude that

$$
u \in \mathcal{G}(\Omega,(a, b))=\{(z, w) \mapsto v(z w): v \in \mathcal{G}(\mathbb{D}, a b)\}, \quad(a, b) \in \Omega \backslash\{(0,0)\} .
$$

The set $\mathcal{G}(\Omega,(0,0))$ is slightly different. We claim that

$$
\mathcal{G}(\Omega,(0,0))=\left\{(z, w) \mapsto \frac{1}{2} v(z w): \quad v \in \mathcal{G}(\mathbb{D}, 0)\right\}
$$

The inclusion " $\supset$ " is obvious. The opposite inclusion follows from the fact that if $u \in \mathcal{G}(\Omega,(0,0))$, then $u$ is constant on the sets $\{(z, w): z w=c\}$, for all $c \in \mathbb{D}$, and the function

$$
v(\lambda)=\max \left\{2 u(\eta, \eta): \eta^{2}=\lambda\right\}, \quad \lambda \in \mathbb{D},
$$

is subharmonic.

Let $\Omega$ be a strictly convex bounded domain in $\mathbb{C}^{n}$ and let $a \in \Omega$. Lempert [L1] has proved that one can construct a function $u$ with the following properties:

$$
\left\{\begin{array}{l}
u \in \mathcal{C}^{\infty}(\Omega \backslash\{a\}) \cap \mathcal{G}(\Omega, a) \\
\left(d d^{c} u\right)^{n} \equiv 0 \quad \text { in } \Omega \backslash\{a\} \\
u(z) \longrightarrow 0 \quad \text { as } z \longrightarrow w \in \partial \Omega
\end{array}\right.
$$

(See the next section for more information about the operator $\left(d d^{c}\right)^{n}$.) In the one dimensional case, the function $-u$ coincides with the classical Green function for $\Omega$ with pole at $a$. Although Lempert's construction cannot be used in the case of more general classes of open sets in $\mathbb{C}^{n}$, one can approach the problem of generalizing Green functions from a different direction. Let $\Omega$ be a connected open subset of $\mathbb{C}^{n}$, and let $a$ be a point in $\Omega$. One defines (see [K1]) the pluricomplex Green function of $\Omega$ with pole at a by the formula

$$
g_{\Omega}(z, a)=\sup \{u(z): u \in \mathcal{G}(\Omega, a)\} .
$$

EXAMPLE 2.2. If $\|\cdot\|$ is a norm in $\mathbb{C}^{n}$ and $B$ is the open unit ball with respect to this norm, then $g_{B}(z, 0)=\log \|z\|$ for $z \in B$. If $\Omega$ is a domain in $\mathbb{C}^{n}$ and $\Phi: \Omega \longrightarrow B$ is a biholomorphic mapping, then $g_{\Omega}\left(z, \Phi^{-1}(0)\right)=\log \|\Phi(z)\|$ for $z \in \Omega$.

EXAMPLE 2.3. If $\Omega$ is as in Example 2.1, then

$$
g_{\Omega}((z, w),(a, b))= \begin{cases}\frac{1}{2} \log |z w| & \text { if }(z, w) \in \Omega,(a, b)=(0,0), \\ \log \left|\frac{z w-a b}{1-\bar{a} \bar{b} z w}\right| & \text { if }(z, w) \in \Omega,(a, b) \in \Omega \backslash\{(0,0)\} .\end{cases}
$$

Note that

$$
g_{\Omega}((z, w),(0,0)) \neq g_{\Omega}((0,0),(z, w)) \quad \text { if } z w \neq 0
$$

For more general examples of this type see [JP2].

Observe that $(z, w) \mapsto g_{\Omega}(z, w)$ is symmetric if and only if it is plurisubharmonic with respect to each variable separately $[\mathrm{C} 1, \mathrm{BD}]$. To overcome this 
problem, Cegrell $[\mathrm{C} 1]$ has introduced a symmetric Green function in $\mathbb{C}^{n}$. Let $\Omega$ be an open subset of $\mathbb{C}^{n}$. Cegrell's function $W_{\Omega}$ is defined by the formula:

$$
W_{\Omega}(z, w)=\sup \{u(z, w)\}, \quad(z, w) \in \Omega \times \Omega,
$$

where the supremum is taken over all functions $u \in \mathcal{S H}(\Omega \times \Omega,[-\infty, 0])$ satisfying the following conditions:

(i) for each $z \in \Omega,\{w \mapsto u(z, w)\} \in \mathcal{P} \mathcal{S H}(\Omega)$,

(ii) for each $w \in \Omega,\{z \mapsto u(z, w)\} \in \mathcal{P} \mathcal{S H}(\Omega)$,

(iii) $u(z, w) \leq \log \|z-w\|-\log \max \left\{\operatorname{dist}\left(z, \mathbb{C}^{n} \backslash \Omega\right), \operatorname{dist}\left(w, \mathbb{C}^{n} \backslash \Omega\right)\right\}$.

Of course, if $g_{\Omega}$ is symmetric, then it coincides with $W_{\Omega}$. In general, $W_{\Omega} \leq g_{\Omega}$, the function $W_{\Omega}$ is symmetric and it shares a number of properties with $g_{\Omega}$, but also displays significant differences (see [C1, C2] for details).

Some fundamental properties of the pluricomplex Green function $g_{\Omega}$ are gathered in the following theorem. Recall that an open bounded and connected set $\Omega \subset \mathbb{C}^{n}$ is said to be hyperconvex if there exists a continuous plurisubharmonic function $\varrho: \Omega \longrightarrow(-\infty, 0)$ such that for each $c \in(-\infty, 0)$ the set $\{z \in \Omega: u(z)<c\}$ is relatively compact in $\Omega$.

Theorem 2.3 Suppose that $\Omega, \Omega^{\prime}$ are domains in $\mathbb{C}^{n}$ and $\mathbb{C}^{m}$, respectively.

(i) If $n=1$ and $\Omega$ is hyperconvex, then $-g_{\Omega}(\cdot, a)$ is the classical Green function for $\Omega$ with pole at a (see $[\mathrm{K} 1]$ ).

(ii) If $z, w \in \Omega, m=n$ and $\Omega \subset \Omega^{\prime}$, then $g_{\Omega}(z, w) \geq g_{\Omega^{\prime}}(z, w)$ (see [K1]).

(iii) If $z, w \in \Omega, m=n, \Omega \subset \Omega^{\prime}$, and $\Omega^{\prime} \backslash \Omega$ is pluripolar, then $g_{\Omega}(z, w)=$ $g_{\Omega^{\prime}}(z, w)$ (see $\left.[\mathrm{K} 1]\right)$.

(iv) If $R>r>0$ and $B(w, r) \subset \Omega \subset B(w, R)$, then for $z \in B(w, r)$ we have

$$
\log (\|z-w\| / R) \leq g_{\Omega}(z, w) \leq \log (\|z-w\| / r),
$$

where $B(a, \varrho)$ denotes the open ball with centre at a and radius $\varrho$ (see $[\mathrm{K} 1]$ ).

(v) If $\Omega$ is bounded and $w \in \Omega$, then $\left\{z \mapsto g_{\Omega}(z, w)\right\} \in \mathcal{G}(\Omega, w)$ (see $[\mathrm{K} 1]$ ).

(vi) If $f: \Omega \longrightarrow \Omega^{\prime}$ is holomorphic, then

$$
g_{\Omega^{\prime}}(f(z), f(w)) \leq g_{\Omega}(z, w), \quad z \in \Omega
$$

(see $[\mathrm{K} 1])$. then

(vii) If $\left(\Omega_{j}\right)_{j \in \mathbf{N}}$ is an increasing sequence of domains in $\mathbb{C}^{n}$ and $\Omega=\bigcup \Omega_{j}$,

$$
g_{\Omega}(z, w)=\lim _{j \rightarrow \infty} g_{\Omega_{j}}(z, w), \quad z \in \Omega, w \in \Omega_{1}
$$

(see $[\mathrm{A} 2, \mathrm{D} 2])$.

(viii) If $\Omega$ is hyperconvex, then the pluricomplex Green function $g_{\Omega}: \bar{\Omega} \times \Omega \longrightarrow$ $[-\infty, 0]$ is continuous (where $\left.g_{\Omega} \mid(\partial \Omega \times \Omega) \equiv 0\right)$ (see [D2]).

(ix) If $\Omega$ is a pseudoconvex domain, then the function $g_{\Omega}: \Omega \times \Omega \longrightarrow[-\infty, 0]$ is upper semicontinuous (see [K2]).

(x) Let $C_{\Omega}$ denote the Carathéodory pseudodistance in $\Omega$ and let $\varrho$ be the Poincaré distance in $\mathbb{D}$. Let $\delta_{\Omega}(z, w)=\inf \{\varrho(\xi, \eta)\}$, where the infimum is taken 
over all $\xi, \eta \in \mathbb{D}$ for which there is a holomorphic mapping $f: \mathbb{D} \longrightarrow \Omega$ such that $f(\xi)=z$ and $f(\eta)=w$. Then $\log \tanh C_{\Omega} \leq g_{\Omega} \leq \log \tanh \delta_{\Omega}$ in $\Omega \times \Omega$ (see $[\mathrm{K} 1])$.

(xi) Define

$$
\sigma(z, w)=\inf \left\{\sum_{j=1}^{m} \tanh ^{-1} \exp \left(\max \left\{g_{\Omega}\left(a_{j}, a_{j+1}\right), g_{\Omega}\left(a_{j+1}, a_{j}\right)\right\}\right)\right\},
$$

where the infimum is taken over all finite chains of points $a_{1}, \ldots, a_{m+1}$ in $\Omega$ such that $a_{1}=z$ and $a_{m+1}=w$. The $\left\{\Omega \mapsto \sigma_{\Omega}\right\}$ is a Schwarz-Pick system. In particular $C_{\Omega} \leq \sigma_{\Omega} \leq K_{\Omega}$, where $K_{\Omega}$ denotes the Kobayashi pseudodistance in $\Omega$ (see $[\mathrm{K} 1])$.

(xii) For $(p, v) \in \Omega \times \mathbb{C}^{n}$ define

$$
A_{\Omega}(p, v)=\limsup _{\substack{\lambda \rightarrow 0 \\ \lambda \neq 0}} \frac{\exp \left(g_{\Omega}((p+\lambda v), p)\right)}{|\lambda|} .
$$

Then $A_{\Omega}$ is an infinitesimal pseudometric in $\Omega$; (see [A1, A2]).

In order to gain a better understanding of the pluricomplex Green functions we will need some information concerning maximal plurisubharmonic functions and the complex Monge-Ampère operator. A discussion of these notions is the objective of the next section.

3. The complex Monge-Ampère operator and maximality. Let $\Omega$ be an open subset of $\mathbb{C}^{n}$ and let $u: \Omega \longrightarrow[-\infty, \infty)$ be a plurisubharmonic function. Following Sadullaev $[\mathrm{S}]$, we say that $u$ is maximal if for every relatively compact open subset $G$ of $\Omega$, and for every upper semicontinuous function $v$ on $\bar{G}$ such that $v \in \mathcal{P S H}(G)$ and $v \leq u$ on $\partial G$, we have $v \leq u$ in $G$. We shall be using the symbol $\mathcal{M P S H}(\Omega)$ to denote the family of all maximal plurisubharmonic functions on $\Omega$. Note that maximal plurisubharmonic functions may be unbounded. For instance $\{(z, w) \mapsto \log |z w|\} \in \mathcal{M P S H}\left(\mathbb{C}^{2}\right)$.

It follows directly from the definition that if $u \in \mathcal{M P S H}(\Omega)$ and $v \in \mathcal{P H}(\Omega)$ (where $\mathcal{P H}=$ pluriharmonic), then $v, u+v \in \mathcal{M P S H}(\Omega)$. Moreover, the limit of a locally uniformly convergent sequence of maximal plurisubharmonic functions is a maximal plurisubharmonic function.

It is convenient to keep in mind analogies to convex and subharmonic functions. In one real variable, affine functions are the "maximal" convex functions. In several real variables, harmonic functions can be regarded as maximal subharmonic functions. The maximality of convex functions of several real variables is somewhat more involved (see e.g. [RT]). Since convex functions of many variables can be described as "pluri-sub-affine", there is a significant similarity to the plurisubharmonic case which we describe below.

Although the maximal psh functions of one complex variable are $\mathcal{C}^{\infty}$, in higher dimensions it is easy to find non-differentiable or even discontinuous maximal 
plurisubharmonic functions. One-dimensional potential theory draws much of its strength from the fact that harmonic functions are smooth and can be characterized in terms of the Laplace operator. In the case of $\mathcal{C}^{2}$-plurisubharmonic functions, the complex Hesse matrix $\left[\frac{\partial^{2} u}{\partial z_{j} \partial \bar{z}_{k}}\right]$ carries information about maximality of $u$. We have the following (see e.g. [K3])

Proposition 3.1. Let $u \in \mathcal{C}^{2}(\Omega)$, where $\Omega \subset \mathbb{C}^{n}$ is open. Then $u \in \mathcal{M P S H}(\Omega)$ if and only if $\operatorname{det}\left[\frac{\partial^{2} u}{\partial z_{j} \partial \bar{z}_{k}}\right] \equiv 0$ in $\Omega$.

If $\partial$ and $\bar{\partial}$ are the standard differential operators in $\mathbb{C}^{n}$, then we define the operator $d^{c}$ by the formula $d^{c}=\mathrm{i}(\bar{\partial}-\partial)$. If $d$ denotes the operator of exterior differentiation,we have $d d^{c}=2 \mathrm{i} \partial \bar{\partial}$. The complex Monge-Ampère operator in $\mathbb{C}^{n}$ is defined as the $n$th exterior power of $d d^{c}$, i.e.

$$
\left(d d^{c}\right)^{n}=\underbrace{d d^{c} \wedge \ldots \wedge d d^{c}}_{n-\text { times }} .
$$

Observe that if $u \in \mathcal{C}^{2}(\omega)$, then

$$
\left(d d^{c} u\right)^{n}=4^{n} n ! \operatorname{det}\left[\frac{\partial^{2} u}{\partial z_{j} \partial \bar{z}_{k}}\right] d V
$$

where $d V$ denotes the usual volume form in $\mathbb{C}^{n}$.

Now the above proposition can be restated as follows.

Corollary 3.2. Let $\Omega$ be an open subset of $\mathbb{C}^{n}$, and let $u \in \mathcal{C}^{2} \cap \mathcal{P S H}(\Omega)$. Then $u$ is maximal if and only if $\left(d d^{c} u\right)^{n}=0$ in $\Omega$.

The next proposition and theorem provide some examples of maximal plurisubharmonic functions.

Let $f: \Omega \longrightarrow \Omega^{\prime}$ be a holomorphic mapping, where $\Omega, \Omega^{\prime} \subset \mathbb{C}^{n}$, and let $u \in \mathcal{C}^{2}\left(\Omega^{\prime}\right)$. It is easy to check that

$$
\operatorname{det}\left[\frac{\partial^{2}(u \circ f)}{\partial z_{j} \partial \bar{z}_{k}}(a)\right]=\left|\operatorname{det} \partial_{a} f\right|^{2} \operatorname{det}\left[\frac{\partial^{2} u}{\partial w_{j} \partial \bar{w}_{k}}(f(a))\right] \text {. }
$$

Consequently, we obtain the following transformation formula for the MongeAmpère operator:

$$
\left(d d^{c}(u \circ f)\right)^{n}(a)=\left|\operatorname{det} \partial_{a} f\right|^{2}\left(d d^{c} u\right)^{n}(f(a)) .
$$

Proposition 3.3. Let $\Omega$ be an open subset of $\mathbb{C}^{n}$. If $u_{1}, \ldots, u_{m} \in \mathcal{P H}(\Omega)$ and $m \leq n$, then $\max \left\{u_{1}, \ldots, u_{m}\right\} \in \mathcal{M P S H}(\Omega)$.

Proof. Since the functions $u_{1}, \ldots, u_{m}$ do not have to be all different, we may suppose that $m=n$. Moreover, since for locally bounded plurisubharmonic functions the property of being a maximal plurisubharmonic function is local (see below), we may suppose that there are holomorphic functions $f_{1}, \ldots, f_{n}$ such that $u_{j}=\operatorname{Re} f_{j}$ in $\Omega$ for all $j$. Let $v=\max \left\{u_{1}, \ldots, u_{n}\right\}$. Define

$$
F_{p}=\left(\exp \left(p f_{1}\right), \ldots, \exp \left(p f_{n}\right)\right), \quad p=1,2,3, \ldots
$$


We have

$$
v(z)=\log \max \left\{\left|\exp \left(f_{1}\right)\right|, \ldots,\left|\exp \left(f_{n}\right)\right|\right\} \leq \frac{1}{p} \log \left\|F_{p}(z)\right\| \leq \frac{1}{2 p} \log n+v(z)
$$

in $\Omega$. Since $z \mapsto \log \|z\|$ is maximal in $\mathbb{C}^{n} \backslash\{0\},(3.1)$ implies that $z \mapsto \log \left\|F_{p}(z)\right\|$ is maximal in $\Omega$ for each $p$. Therefore $v$ is maximal as the limit of a uniformly convergent sequence of maximal functions.

This statement is no longer true if $m>n$. For instance, the function

$$
v(z)=\log \max \left\{1,\left|z_{1}\right|, \ldots,\left|z_{n}\right|\right\}, \quad z \in \mathbb{C}^{n},
$$

is obviously not maximal in $\mathbb{C}^{n}$.

Although the function defined as the maximum of two maximal functions is not necessarily maximal, we have the following result due to Zeriahi [Z].

THEOREM 3.4. Let $\Omega_{1}$ and $\Omega_{2}$ be open sets in $\mathbb{C}^{n_{1}}$ and $\mathbb{C}^{n_{2}}$, respectively. Suppose that $u_{1} \in \mathcal{M P S H}\left(\Omega_{1}\right)$ and $u_{2} \in \mathcal{M P S H}\left(\Omega_{2}\right)$. If $u\left(z_{1}, z_{2}\right)=\max \left\{u_{1}\left(z_{1}\right)\right.$, $\left.u_{2}\left(z_{2}\right)\right\}$ for $\left(z_{1}, z_{2}\right) \in \Omega_{1} \times \Omega_{2}$, then $u \in \mathcal{M P S H}\left(\Omega_{1} \times \Omega_{2}\right)$.

The characterization of maximality given in Corollary 3.2 can be extended to the case of locally bounded plurisubharmonic functions. First, however, it is necessary to generalize the complex Monge-Ampère operator so that it can be applied to such functions. The starting point is the following property [CLN, BT1].

Proposition 3.5. Let $\Omega$ be an open subset of $\mathbb{C}^{n}$. If $v^{1}, \ldots, v^{k} \in \mathcal{C}^{2}(\Omega)$ and $\varphi$ is a test form in $\Omega$ of bidegree $(n-k, n-k)$, then

$$
\begin{array}{r}
\int_{\Omega} d d^{c} v^{1} \wedge \ldots \wedge d d^{c} v^{k} \wedge \varphi=\int_{\Omega} v^{j} d d^{c} v^{1} \wedge \ldots \wedge d d^{c} v^{j-1} \wedge d d^{c} v^{j+1} \wedge \ldots \\
\ldots \wedge d d^{c} v^{k} \wedge d d^{c} \varphi
\end{array}
$$

for $j=1, \ldots, k$.

The above proposition, combined with some facts concerning positive differential forms, can be used to obtain the following inequality.

Proposition 3.6. Let $\Omega$ be an open neighbourhood of a compact set $K \subset \mathbb{C}^{n}$. There exist a constant $C>0$ and a compact set $L \subset \Omega \backslash K$, which depend on $K$ and $\Omega$, such that for all $u_{1}, \ldots, u_{n} \in \mathcal{P S H} \cap \mathcal{C}^{2}(\Omega)$,

$$
\int_{K} d d^{c} u_{1} \wedge \ldots \wedge d d^{c} u_{n} \leq C\left\|u_{1}\right\|_{L} \cdot \ldots \cdot\left\|u_{n}\right\|_{L},
$$

where $\|\cdot\|_{L}$ denotes the supremum norm on $L$.

This is a slightly enhanced version of the the Chern-Levine-Nirenberg estimate ([CLN]; see also [K3]).

If $u \in \mathcal{P S H}(\Omega)$, then $d d^{c} u$ is a positive $(1,1)$-current. Bedford and Taylor [BT1] observed that this property, combined with the integration-by-part formula from Proposition 3.5, can serve as a basis for an inductive definition of the 
Monge-Ampère operator acting on locally bounded plurisubharmonic functions. Let $u^{1}, \ldots, u^{n} \in L_{\text {loc }}^{\infty}(\Omega) \cap \mathcal{P S H}(\Omega)$. If $1 \leq k \leq n$, then $d d^{c} u^{1} \wedge \ldots \wedge d d^{c} u^{k}$ can be defined inductively, as a positive $(k, k)$-current of order 0 , by the formula

$$
\int_{\Omega} d d^{c} u^{1} \wedge \ldots \wedge d d^{c} u^{k} \wedge \chi=\int_{\Omega} u^{k} d d^{c} u^{1} \wedge \ldots \wedge d d^{c} u^{k-1} \wedge d d^{c} \chi,
$$

where $\chi$ is a test form in $\Omega$ of bidegree $(n-k, n-k)$.

The operator $\left(d d^{c}\right)^{n}$, acting on locally bounded plurisubharmonic functions, is called the generalized complex Monge-Ampère operator. One of the most important properties of the Monge-Ampère operator is continuity on monotone sequences. As every plurisubharmonic function can be approximated by a decreasing sequence of smooth plurisubharmonic functions, algebraic properties of the Monge-Ampère operator acting on smooth functions are retained in the general case [BT2].

THEOREM 3.7. Let $\Omega$ be an open subset of $\mathbb{C}^{n}$, and let $\left\{u_{j}\right\}_{j \in \mathbf{N}}$ be a monotone sequence in $\mathcal{P S H} \cap L_{\text {loc }}^{\infty}(\Omega)$ that converges to a function $u \in \mathcal{P S H} \cap L_{\text {loc }}^{\infty}(\Omega)$ almost everywhere in $\Omega$ (with respect to the Lebesgue measure). Then the sequence of the Radon measures $\left(d d^{c} u_{j}\right)^{n}$ converges to the measure $\left(d d^{c} u\right)^{n}$ in the weak $k^{*}$-topology.

This leads, via some comparison results for plurisubharmonic functions, to the following characterization of maximality [BT2, S, K3].

THeOREM 3.8. Let $\Omega$ be an open subset of $\mathbb{C}^{n}$, and let $u$ be a locally bounded plurisubharmonic function on $\Omega$. Then $u$ is maximal if and only if it satisfies the homogeneous Monge-Ampère equation $\left(d d^{c} u\right)^{n}=0$.

It is natural to ask whether the above criterion of maximality extends to some unbounded functions. The next theorem contains a positive result in this direction $([\mathrm{S}],[\mathrm{BE}])$.

THEOREM 3.9. Let $\Omega$ be an open subset of $\mathbb{C}^{n}$ and let $\left\{u_{j}\right\}_{j \in \mathbf{N}}$ be a decreasing sequence in $\mathcal{P S H} \cap L_{\mathrm{loc}}^{\infty}(\Omega)$ that converges to a function $u \in \mathcal{P S H}(\Omega)$. If the sequence of the Radon measures $\left(d d^{c} u_{j}\right)^{n}$ converges to zero in the weak $k^{*}$-topology, then $u \in \mathcal{M P S H}(\Omega)$.

There are several methods of defining the complex Monge-Ampère operator as a positive measure for some unbounded plurisubharmonic functions (see e.g. $[\mathrm{K}, \mathrm{D} 1, \mathrm{SI}, \mathrm{C} 1, \mathrm{~B}]$.$) In the present context, we need a very simple special case of$ this kind of definition, so that logarithmic poles can be handled.

Let $\Omega$ be an open connected subset of $\mathbb{C}^{n}$, and let $a$ be a point in $\Omega$. Define:

$$
\mathcal{P S H}(\Omega ; a)=\mathcal{P S H}(\Omega) \cap L_{\text {loc }}^{\infty}(\Omega \backslash\{a\}) .
$$

By using Proposition 3.5 and the Chern-Levine-Nirenberg estimate (Proposition 3.6) one can prove the following property.

Proposition 3.10. Let $\Omega$ be an open set in $\mathbb{C}^{n}$, and let $u \in \mathcal{P S H}(\Omega ; a)$. Then there exists a positive Borel measure $\mu$ on $\Omega$ such that, for any decreasing 
sequence $\left\{u_{j}\right\}_{j \in f N} \subset \mathcal{P S H} \cap L_{\mathrm{loc}}^{\infty}(\Omega)$ convergent to $u$ at each point in $\Omega$, the sequence $i g\left\{\left(d d^{c} u_{j}\right)^{n} i g\right\}_{j \in \mathbf{N}}$ is weak $k^{*}$-convergent to $\mu$.

If $u \in \mathcal{P} \mathcal{S H} \cap L_{\text {loc }}^{\infty}(\Omega)$, then the measure $\mu$ from the above proposition coincides with $\left(d d^{c} u\right)^{n}$; this is why for $u \in \mathcal{P} \mathcal{S H}(\Omega ; a)$ we put $\left(d d^{c} u\right)^{n}=\mu$.

ExAmple 3.11. Let $a \in \mathbb{C}^{n}$, and let $R>0$. If $u(z)=\log (\|z-a\| / R)$ for all $z \in \mathbb{C}^{n}$, then $\left(d d^{c} u\right)^{n}=(2 \pi)^{n} \delta_{a}$, where $\delta_{a}$ is the Dirac delta function at $a$.

The next property, which is a special case of a more general theorem given by Demailly [D1, D2], will be needed in the next section.

TheOREM 3.12. Let $\Omega$ be a bounded domain in $\mathbb{C}^{n}$, let $a \in \Omega$, and let $u, v \in$ $\mathcal{P S H} \cap \mathcal{C}(\Omega,[-\infty, \infty))$ be such that $u^{-1}(-\infty)=v^{-1}(-\infty)=\{a\}, u<v$ in $\Omega \backslash\{a\}$, and

Then $\left(d d^{c} u\right)^{n}(\{a\}) \leq\left(d d^{c} v\right)^{n}(\{a\})$.

$$
\limsup _{z \rightarrow a} \frac{u(z)}{v(z)}=1
$$

4. Green functions and the complex Monge-Ampère operator. It is not difficult to check (see $[\mathrm{K} 1, \mathrm{~K} 3]$ ) that if $\Omega$ is bounded, then $z \mapsto g_{\Omega}(z, w)$ is maximal in $\Omega \backslash\{w\}$. Consequently,

$$
\left(d d^{c} g_{\Omega}(\cdot, w)\right)^{n} \equiv 0 \quad \text { in } \Omega \backslash\{w\} .
$$

In fact, it turns out that in hyperconvex domains the pluricomplex Green function can be fully characterized in terms of a Dirichlet problem for the Monge-Ampère operator. Let $\Omega$ be a bounded domain in $\mathbb{C}^{n}$, and let $a \in \Omega$. Consider the problem of finding a function $u$ which satisfies the following conditions:

$$
\left\{\begin{array}{l}
u \in \mathcal{C}(\Omega \backslash\{a\}) \cap \mathcal{G}(\Omega, a), \\
\left(d d^{c} u\right)^{n}=(2 \pi)^{n} \delta_{a} \text { in } \Omega \\
u(z) \rightarrow 0 \text { as } z \rightarrow \partial \Omega,
\end{array}\right.
$$

where $\delta_{a}$ is the Dirac delta function at $a$.

We have the following theorem due to Demailly [D2] (see also [L1]).

THEOREM 4.1. If $\Omega$ is hyperconvex, then the function $u(z)=g_{\Omega}(z, a)$ is a unique solution to the problem (4.1).

It should be noted that, in general, the pluricomplex Green function does not have to be very smooth. For instance, if $P(0,1) \subset \mathbb{C}^{n+1}$ is the unit polydisc, then the function $z \mapsto g_{P(0,1)}(z, 0)$ is not differentiable. Bedford and Demailly [BD] have shown that even if $\Omega$ is strictly pseudoconvex with a $\mathcal{C}^{2}$-boundary, $g_{\Omega}$ is not necessarily a $\mathcal{C}^{1}$-function (with respect to the first variable).

In Section 2 we described the symmetric pluricomplex Green function $W_{\Omega}$ introduced by Cegrell. It should be mentioned that the situation in which $W_{\Omega}$ and $g_{\Omega}$ coincide can be characterized in terms of the Monge-Ampère operator (see $[\mathrm{C} 1, \mathrm{C} 2])$. 
We are going to describe just one application of Theorem 4.1. Because of the close relationship between the pluricomplex Green function and the Carathéodory and Kobayashi pseudodistances, it is reasonable to expect that the Green function has the product property enjoyed by these pseudometrics. The following theorem due to Jarnicki and Pflug [JP2] shows that this is indeed the case under the assumption of pseudoconvexity. The short proof presented here is slightly different from that given in [JP2], although both proofs use Zeriahi's result from the previous section.

THEOREM 4.2. Let $\Omega_{1}$ and $\Omega_{2}$ be pseudoconvex domains in $\mathbb{C}^{n_{1}}$ and $\mathbb{C}^{n_{2}}$, respectively, and let $\Omega=\Omega_{1} \times \Omega_{2}$. Then

$$
g_{\Omega}\left(\left(w_{1}, w_{2}\right),\left(z_{1}, z_{2}\right)\right)=\max \left\{g_{\Omega_{1}}\left(w_{1}, z_{1}\right), g_{\Omega_{2}}\left(w_{2}, z_{2}\right)\right\}
$$

for all $\left(w_{1}, z_{1}\right),\left(w_{2}, z_{2}\right) \in \Omega$.

P r o of. First suppose that both $\Omega_{1}$ and $\Omega_{2}$ are hyperconvex. Fix $z=\left(z_{1}, z_{2}\right)$ $\in \Omega$. Define

$$
g(w)=\max \left\{g_{\Omega_{1}}\left(w_{1}, z_{1}\right), g_{\Omega_{2}}\left(w_{2}, z_{2}\right)\right\}, \quad w=\left(w_{1}, w_{2}\right) \in \Omega .
$$

In view of Theorem 2.3(viii), the function $g \mid \Omega \backslash\left\{\left(z_{1}, z_{2}\right)\right\}$ is continuous. Furthermore, there are positive constants $A$ and $B$ such that

$$
\log \|w-z\|+A<g(w)<\log \|w-z\|+B
$$

for all $w \neq z$ which are sufficiently close to $z$ (see Proposition 6.11 in [K2]). Therefore, by Demailly's comparison principle (Theorem 3.12), we have:

$$
\left(d d^{c} g\right)^{n_{1}+n_{2}}(\{z\})=(2 \pi)^{n_{1}+n_{2}} .
$$

By Theorem 3.4 above and Proposition 4.6.4 in [K3],

$$
\left(d d^{c} g\right)^{n_{1}+n_{2}}=0 \quad \text { in } \Omega \backslash\left(w_{1}, w_{2}\right) .
$$

Thus $g$ is a solution to the Dirichlet problem

$$
\left\{\begin{array}{l}
g \in \mathcal{C}(\Omega \backslash\{z\}) \cap \mathcal{G}(\Omega, z) \\
\left(d d^{c} g\right)^{n_{1}+n_{2}}=(2 \pi)^{n_{1}+n_{2}} \delta_{z} \quad \text { in } \Omega \\
g(w) \rightarrow 0 \quad \text { as } w \rightarrow \partial \Omega
\end{array}\right.
$$

Consequently $g$ is the pluricomplex Green function for $\Omega$ with pole at $z$ by Theorem 4.1. The general case follows from Theorem 2.3(vii).

Corollary 4.3. If $\Omega_{1}, \ldots, \Omega_{k}$ are bounded domains in the complex plane which are regular with respect to the classical Dirichlet problem and $\Omega=\Omega_{1} \times$ $\ldots \times \Omega_{k}$, then

$$
g_{\Omega}(w, z)=g_{\Omega}(z, w)=\max _{j=1, \ldots, k}\left\{g_{\Omega_{j}}\left(w_{j}, z_{j}\right)\right\},
$$

for all $z=\left(z_{1}, \ldots, z_{k}\right)$ and $w=\left(w_{1}, \ldots, w_{k}\right)$ in $\Omega$.

EXAMPLE 4.4. Let

$$
\Omega=\left\{\left(z_{1}, \ldots, z_{n}\right) \in \mathbb{C}^{n}: \operatorname{Im} z_{j} \in(0,1), j=1, \ldots, n\right\} .
$$


Then

$$
g_{\Omega}(z, w)=\frac{1}{2} \max _{j=1, \ldots, n}\left(\frac{\cosh \left(\pi\left(x_{j}-s_{j}\right)\right)-\cos \left(\pi\left(y_{j}+t_{j}\right)\right)}{\cosh \left(\pi\left(x_{j}-s_{j}\right)\right)-\cos \left(\pi\left(y_{j}-t_{j}\right)\right)}\right),
$$

where $z, w \in \Omega, z_{j}=x_{j}+i y_{j}$, and $w_{j}=s_{j}+i t_{j}$. The one dimensional case is covered in $[\mathrm{BR}]$; the general case follows from the above corollary.

5. Examples of pluricomplex Green functions. Some examples of pluricomplex Green functions can be derived from the following property.

Proposition 5.1. Suppose that $h: \mathbb{C}^{n} \longrightarrow[0, \infty)$ is homogeneous (i.e. $h(\xi z)$ $=|\xi| h(z)$ for all $z \in \mathbb{C}^{n}$ and $\left.\xi \in \mathbb{C}\right)$ and $\log h \in \mathcal{P S H}\left(\mathbb{C}^{n}\right)$. If $\Omega=\left\{z \in \mathbb{C}^{n}\right.$ : $h(z)<1\}$, then

$$
g_{\Omega}(z, 0)=\log h(z), \quad z \in \Omega .
$$

Proof. As in the proof of Lemma 6.1.3 in [K3] we check that $u \leq \log h$ in $\Omega \backslash h^{-1}(0)$ and, since $h^{-1}(0)$ is pluripolar, it follows that $u \leq \log h$ in $\Omega$, as required.

It can be shown that the pluricomplex Green functions are invariant with respect to a class of proper holomorphic mappings, which includes all biholomorphic mappings (see Theorem 6.1.6 in [K3]). This property, combined with the above proposition, can be put to use as follows.

Proposition 5.2. Let $p \in \mathbf{N}$, and let $\mathbf{M}_{n}$ denote the vector space of all complex $n \times n$-matrices. Let $F$ be a complex polynomial mapping from $\mathbb{C}^{n}$ to $\mathbf{M}_{n}$ such that $\operatorname{det} F(z) \equiv$ const.$\neq 0$ and $(n-1) \operatorname{deg} F<p$. Suppose that $h$ is a non-negative homogeneous function on $\mathbb{C}^{n}$ such that $\log h \in \mathcal{P} \mathcal{S H}\left(\mathbb{C}^{n}\right)$. If

$$
f(z)=F(z) \cdot\left[\begin{array}{c}
z_{1}^{p} \\
\vdots \\
z_{n}^{p}
\end{array}\right], \quad z=\left(z_{1}, \ldots, z_{n}\right) \in \mathbb{C}^{n},
$$

then $f$ is proper and

$$
g_{\Omega}(z, 0)=\frac{1}{p} \log h(f(z))
$$

for all $z \in \Omega$, where $\Omega=\left\{z \in \mathbb{C}^{n}: h(f(z))<1\right\}$.

Proof. Suppose that $\mathbf{M}_{n}$ is endowed with the operator norm corresponding to the polydisc norm in $\mathbb{C}^{n}$. Note that

$$
\left|F(z)^{-1}\right|^{-1} \leq \frac{|f(z)|}{|z|^{p}} \leq|F(z)|, \quad z \neq 0 .
$$

Therefore, since $\operatorname{deg}\left\{z \mapsto F(z)^{-1}\right\} \leq(n-1) \operatorname{deg} F$, we have $|f(z)| \geq|z|^{p}\left|F(z)^{-1}\right|^{-1}$ $\rightarrow \infty$ as $|z| \rightarrow \infty$. To complete the proof it is enough to use Proposition 5.1 above and Theorem 6.1.6 in [K3].

EXAMPLE 5.3. Let $d$ be a positive integer and let $f_{j k}: \mathbb{C}^{n} \longrightarrow \mathbb{C}$ be a polynomial of degree at most $d$ for $j=2, \ldots, n$ and $k=1, \ldots, j-1$. Let 
$a_{1}, \ldots, a_{n} \in \mathbb{C} \backslash\{0\}$. Define

$$
f\left(z_{1}, \ldots, z_{n}\right)=\left[\begin{array}{ccccc}
a_{1} & 0 & 0 & \ldots & 0 \\
f_{21}(z) & a_{2} & 0 & \ldots & 0 \\
f_{31}(z) & f_{32}(z) & a_{3} & \ldots & 0 \\
\vdots & \vdots & \vdots & \ldots & \vdots \\
f_{n 1}(z) & f_{n 2}(z) & f_{n 3}(z) & \ldots & a_{n}
\end{array}\right] \cdot\left[\begin{array}{c}
z_{1}^{(d+1)(n-1)} \\
z_{2}^{(d+1)(n-1)} \\
z_{3}^{(d+1)(n-1)} \\
\vdots \\
z_{n}^{(d+1)(n-1)}
\end{array}\right]
$$

for $z=\left(z_{1}, \ldots, z_{n}\right) \in \mathbb{C}^{n}$. Let $\Omega=f^{-1}\left(\mathbb{D}^{n}\right)$. Then, according to Proposition 5.2, we have

for all $z \in \Omega$.

$$
g_{\Omega}(z, 0)=\frac{\log |f(z)|}{(d+1)(n-1)}
$$

In view of Proposition 5.1, if $\Omega$ is a logarithmically convex complete Reinhardt domain, then $g_{\Omega}(z, 0)=\log p(z)$ for $z \in \Omega$, where $p$ denotes the Minkowski functional of $\Omega$; (see [JP1] or [K2]). In [JP1, JP2] the authors obtained explicit formulas for pluricomplex Green functions in some Reinhardt domains. Their approach was based on Hadamard's Three Circles Theorem for logarithmically subharmonic functions of one complex variable. Hadamard's theorem can be viewed as a result about convexity of certain functions. Moreover, if a function in a Reinhardt domain in $\mathbb{C}^{n}$ depends only on $\left(\left|z_{1}\right|, \ldots,\left|z_{n}\right|\right)$, its plurisubharmonicity can be characterized in terms of convexity of an associated function. Consequently, one could expect that the language of convex functions and sets should provide an ideal environment for such constructions. In the remainder of this section we show that this is indeed the case.

Let

$$
\lambda\left(z_{1}, \ldots, z_{n}\right)=\left(\log \left|z_{1}\right|, \ldots, \log \left|z_{n}\right|\right), \quad\left(z_{1}, \ldots, z_{n}\right) \in \mathbb{C}^{n}
$$

and

$$
e\left(x_{1}, \ldots, x_{n}\right)=\left(e^{x_{1}}, \ldots, e^{x_{n}}\right), \quad\left(x_{1}, \ldots, x_{n}\right) \in[-\infty, \infty)^{n},
$$

where $\log 0=-\infty$ and $e^{-\infty}=0$.

A function $g$ defined on a Reinhardt domain $\Omega$ is called polyradial if $g\left(z_{1}, \ldots\right.$ $\left.\ldots z_{n}\right)=g\left(\left|z_{1}\right|, \ldots,\left|z_{n}\right|\right)$ for all $z \in \Omega$. Note that if $\Omega$ is a Reinhardt domain containing the origin, then the pluricomplex Green function of $\Omega$ with pole at 0 is polyradial, since it is invariant with respect to the biholomorphic transformations of the form $\left(z_{1}, \ldots, z_{n}\right) \mapsto\left(\lambda_{1} z_{1}, \ldots, \lambda_{n} \zeta_{n}\right)$, where $\left(\lambda_{1}, \ldots, \lambda_{n}\right) \in(\partial \mathbb{D})^{n}$ is fixed. Note also that if $\Omega$ is a Reinhardt domain in $\mathbb{C}^{n}$ and $g$ is a negative polyradial function in $\Omega$, then $g \in \mathcal{P S H}(\Omega)$ if and only if the restriction of $g \circ e$ to $\lambda(\Omega) \cap \mathbb{R}^{n}$ is convex.

In order to describe our construction, it is convenient to introduce some more terminology. A ray $(t o-\infty)$ is a half-line of the form $L=\{a+t b: t \leq 0\}$, where $a \in \mathbb{R}^{n}$ and $b \in(0,+\infty)^{n}$. In this case $a$ is called the end-point of $L$. In what 
follows, we put

$$
\max (x)=\max \left\{x_{1}, \ldots, x_{n}\right\}, \quad x=\left(x_{1}, \ldots, x_{n}\right) \in \mathbb{R}^{n},
$$

and we define

$$
\mathcal{M}(c)=\left\{x \in \mathbb{R}^{n}: \max (x)<c\right\}, \quad c \in \mathbb{R} .
$$

Suppose that $\omega \subset \mathbb{R}^{n}$ is a domain such that for some $c \in \mathbb{R}$ we have $\mathcal{M}(c) \subset \omega$. We say that $u: \omega \longrightarrow \mathbb{R}$ has normalized growth at $-\infty$ if for some real number $d$ we have

$$
\sup \{u(x)-\max (x): x \in \omega \cap \mathcal{M}(d)\}<+\infty .
$$

We have the following characterization.

COROLlaRY 5.4. Let $\Omega$ be a connected Reinhardt domain containing the origin and let $g$ be a polyradial plurisubharmonic function on $\Omega$. Then $g$ has a logarithmic pole at 0 if and only if $g \circ e$ has normalized growth at $-\infty$.

Suppose that $\omega \subset \mathbb{R}^{n}$ is a domain such that for some $c_{1}, c_{2} \in \mathbb{R}$ we have $\mathcal{M}\left(c_{1}\right) \subset \omega \subset \mathcal{M}\left(c_{2}\right)$. Define

$$
u_{\omega}(x)=\sup \{u(x)\}, \quad x \in \omega,
$$

where the supremum is taken over all convex functions $u: \omega \longrightarrow(-\infty, 0]$ with normalized growth at $-\infty$.

For any $c \in \mathbb{R}$, we have

$$
u_{\mathcal{M}(c)}(x)=\max (x)-c, \quad x \in \mathcal{M}(c) .
$$

Moreover, $u_{\omega_{1}} \geq u_{\omega_{2}}$ if $\omega_{1} \subset \omega_{2}$. Furthermore, if $\Omega \subset \mathbb{C}^{n}$ is a bounded Reinhardt domain and $\omega=\lambda(\Omega) \cap \mathbb{R}^{n}$, then

$$
\left.u_{\omega}(x)=\left(g_{\Omega}(e(x), 0)\right)\right), \quad x \in \omega .
$$

Our examples will be based on the following two propositions.

Proposition 5.5. If $\omega \subset \mathbb{R}^{n}$ is convex and such that for some $c_{1}, c_{2} \in \mathbb{R}$ we have $\mathcal{M}\left(c_{1}\right) \subset \omega \subset \mathcal{M}\left(c_{2}\right)$, then

$$
\lim _{\substack{z \rightarrow a \\ z \in \omega}} u_{\omega}(z)=0
$$

for all $a \in \partial \omega$. In particular, if $\omega^{\prime} \subset \mathbb{R}^{n}$ is a domain such that $\mathcal{M}\left(c_{1}\right) \subset \omega^{\prime} \subset \omega$ and $a \in \partial \omega \cap \partial \omega^{\prime}$, then $\lim _{z \in \omega^{\prime}}^{z \rightarrow a} u_{\omega^{\prime}}(z)=0$.

Proof. Let $a \in \omega$ and let $L: \mathbb{R}^{n} \longrightarrow \mathbb{R}$ be an affine mapping such that $L(a)=0$ and $L<0$ on $\omega$. Then $L$ is of the form

$$
L\left(\left(x_{1}, \ldots, x_{n}\right)\right)=\sum_{j=1}^{n} c_{j} x_{j}+d, \quad\left(x_{1}, \ldots, x_{n}\right) \in \mathbb{R}^{n},
$$

for some $\left(c_{1}, \ldots, c_{n}\right) \in[0, \infty)^{n}$ and $d \in \mathbb{R}$. For some $c>0$ we have $c L \leq u_{\omega}$ and so the result follows. 
Proposition 5.6. Let $\omega \subset \mathbb{R}^{n}$ be a convex domain such that for some $c_{1}, c_{2} \in$ $\mathbb{R}$ we have $\mathcal{M}\left(c_{1}\right) \subset \omega \subset \mathcal{M}\left(c_{2}\right)$. Suppose that $x_{0} \in \bar{\omega}$ is the end point of a ray $R$ such that $R \backslash\left\{x_{0}\right\}$ is contained in $\omega$. If $x$ belongs to this ray, then

$$
u_{\omega}(x) \leq \max \left(x-x_{0}\right)+u_{\omega}^{*}\left(x_{0}\right),
$$

where

$$
u_{\omega}^{*}\left(x_{0}\right)=\limsup _{\substack{x \rightarrow x_{0} \\ x \in \omega}} u_{\omega}(x) .
$$

Proof. It it enough to verify the estimate for $x_{0} \in \omega, c_{1}<0$ and $x \neq x_{0}$. Then $x_{0}-x \in(0,+\infty)^{n}$ and the ray in question is of the form $R((-\infty, 0])$, where $R(t)=t\left(x_{0}-x\right)+x_{0}$ for $t \in \mathbb{R}$. Let $u$ be an element of the defining family for $u_{\omega}$. Choose $t<0$ such that $R(t) \in \mathcal{M}\left(c_{1}\right)$. Let $s \in[t, 0]$. Since $u \circ R$ is convex, we have

So

$$
\begin{aligned}
u(R(s)) & \leq \frac{u(R(t))-u\left(x_{0}\right)}{t} s+u\left(x_{0}\right) \\
& \leq-s \max \left(\frac{-R(t)}{t}\right)-\frac{c_{1}+u\left(x_{0}\right)}{t}+u\left(x_{0}\right) \\
& \longrightarrow-s \max \left(x-x_{0}\right)+u\left(x_{0}\right) \quad \text { as } t \longrightarrow-\infty .
\end{aligned}
$$

$$
u(x)=u(R(-1)) \leq \max \left(x-x_{0}\right)+u\left(x_{0}\right) .
$$

COROLlary 5.7. The function $u_{\omega}$ is increasing along rays to $-\infty$ contained in $\omega$.

In each of the following examples $\Omega$ is a Reinhardt domain in $\mathbb{C}^{2}$ and $\omega=$ $\lambda(\Omega) \cap \mathbb{R}^{n}$ is its logarithmic image in $\mathbb{R}^{2}$. Propositions 5.5 and 5.6, combined with the convexity of $u_{\omega}$, have been used to derive the formula for $u_{\omega}$ form a diagram of $\omega$. Then one uses $(5.1)$ to obtain a formula for $g_{\Omega}(0,$.$) on a dense subset of \Omega$. In each case the continuous extension to $\Omega$ gives the required Green function.

Example 5.8. Let $A, B, C, D \in(0,1)$ be such that $A>B$ and $C<D$. Define

$$
E=\left\{(x, y) \in[0,1]^{2}: x=A^{t} C^{1-t}, y=B^{t} D^{1-t}, t \in[0,1]\right\}
$$

and

$$
\Omega=\left\{(z, w) \in \mathbb{C}^{2}:|z|<1,|w|<1,(|z|,|w|) \notin E\right\} .
$$

We are going to divide $\Omega$ into four subsets $\Omega_{1}, \Omega_{2}, \Omega_{3}, \Omega_{4}$. We put:

$$
\begin{aligned}
& \Omega_{1}=\left\{(z, w) \in \Omega:|w| \geq \min \left\{\frac{D}{C}|z|,|z|^{\log D / \log C}\right\}\right\} \\
& \Omega_{2}=\left\{(z, w) \in \Omega:|w| \leq \max \left\{\frac{B}{A}|z|,|z|^{\log B / \log A}\right\}\right\} \\
& \Omega_{3}=\{(t z, t w) \in \Omega:(z, w) \in E, t \in(0,1)\} \\
& \Omega_{4}=\Omega \backslash\left(\Omega_{1} \cup \Omega_{2} \cup \Omega_{3}\right) .
\end{aligned}
$$


$g_{\Omega}((z, w),(0,0))$

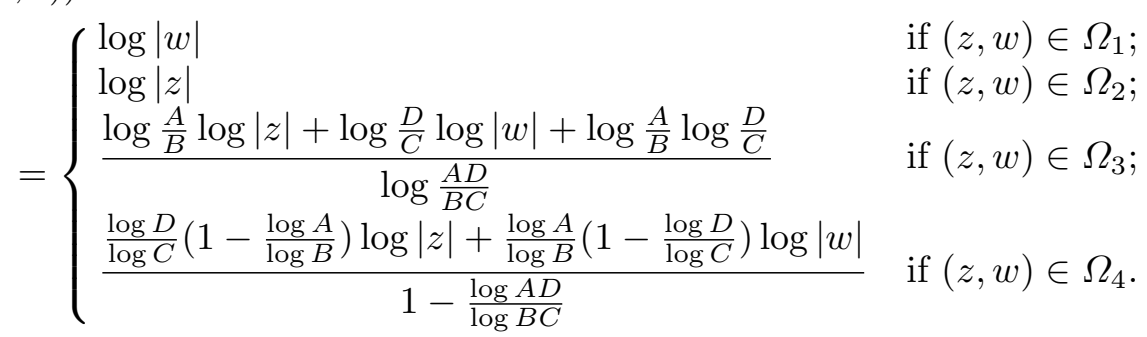

In particular, if $A=C=e^{-2}$ and $B=D=e^{-1}$, then

$\Omega=\left\{(z, w) \in \mathbb{C}^{2}:|z|<1,|w|<1\right\} \backslash\left\{(z, w) \in \mathbb{C}^{2}:|z w| e^{3}=1,|z| \in\left[e^{-2}, e^{-1}\right]\right\}$.

In this case $g_{\Omega}$ is expressed by the following simple formula:

$$
g_{\Omega}((z, w),(0,0))= \begin{cases}\log |w| & \text { if }(z, w) \in \Omega_{1} ; \\ \frac{\log |z|}{\log |z w|} \frac{1}{2}+\frac{1}{2} & \text { if }(z, w) \in \Omega_{2} ; \\ \frac{\log |z w|}{2} & \text { if }(z, w) \in \Omega_{4}\end{cases}
$$

Note that the pluricomplex Green function with pole at the origin for the set $\left\{(z, w) \in \mathbb{C}^{2}:|z|<1,|w|<1\right\} \backslash\left\{(z, w) \in \mathbb{C}^{2}: e^{-2}<|z|<e^{-1}, e^{-2}<|w|<e^{-1}\right\}$ is also given by $(5.2)$.

EXAMPLE 5.9. Let $A, B \in(0,1)$ be such that $A>B$ and let

$$
\Omega=\left\{(z, w) \in \mathbb{C}^{2}:|z|<1,|w|<1\right\} \backslash\left\{(z, w) \in \mathbb{C}^{2}:|z| \leq A,|w|=B\right\} .
$$

It is convenient to divide $\Omega$ into the following subsets:

$$
\begin{aligned}
& \Omega_{1}=\{(z, w) \in \Omega:|z| \leq A, B<|w|\} \\
& \Omega_{2}=\left\{(z, w) \in \Omega: A<|z|,|z|^{\log B / \log A}<|w|\right\} ; \\
& \Omega_{3}=\left\{(z, w) \in \Omega:|w| \leq \max \left\{|z| \frac{B}{A},|z|^{\log B / \log A}\right\}\right\} ; \\
& \Omega_{4}=\left\{(z, w) \in \Omega:|z|<A,|z| \frac{B}{A}<|w|<B\right\} .
\end{aligned}
$$

The pluricomplex Green function for $\Omega$ with pole at the origin is given by the formula:

$$
g_{\Omega}((z, w),(0,0))= \begin{cases}\frac{\log A}{\log B} \log |w| & \text { if }(z, w) \in \Omega_{1} \cup \Omega_{2} ; \\ \log |z| & \text { if }(z, w) \in \Omega_{3} ; \\ \log |w|+\log \frac{A}{B} & \text { if }(z, w) \in \Omega_{4} .\end{cases}
$$

Observe that the restriction of $g_{\Omega}(\cdot, 0)$ to $\Omega_{2} \cup \Omega_{3} \cup \Omega_{4}$ gives the pluricomplex Green function with pole at $(0,0)$ for that set. This was also shown with the help of Hadamard's Three Circles Theorem in [JP2] (see also [JP1]). 
EXAMPLE 5.10. Let $\Omega \subset \mathbb{C}^{2}$ be the convex Reinhardt domain given by the formula $\Omega=\Omega_{1} \cup \Omega_{2} \cup \Omega_{3}$, where

$$
\begin{aligned}
& \Omega_{1}=\left\{(z, w) \in \mathbb{C}^{2}: e^{-1}<|w / z|<e,(\log |z|)^{2}+(\log |w|)^{2}<1\right\}, \\
& \Omega_{2}=\left\{(z, w) \in \mathbb{C}^{2}:|z|<1, e|z| \leq|w|<e\right\}, \\
& \Omega_{3}=\left\{(z, w) \in \mathbb{C}^{2}:|w|<1, e|w| \leq|z|<e\right\} .
\end{aligned}
$$

The pluricomplex Green function of $\Omega$ is given by the following formula:

$$
g_{\Omega}((z, w),(0,0))= \begin{cases}\frac{\log |w z|-\sqrt{2-\left(\log \left|\frac{w}{z}\right|\right)^{2}}}{2} & \text { if }(z, w) \in \Omega_{1} ; \\ \log |w|-1 & \text { if }(z, w) \in \Omega_{2} ; \\ \log |z|-1 & \text { if }(z, w) \in \Omega_{3} .\end{cases}
$$

Let $P(0,1)$ be the open unit polydisc in $\mathbb{C}^{2}$. It is not difficult to notice that the pluricomplex Green function for $\Omega^{\prime}=P(0,1) \cup \Omega_{1}$ is the restriction of $g_{\Omega}(\cdot, 0)$ to $\Omega^{\prime}$.

Since $\Omega$ is convex and balanced, the function $\exp \left(g_{\Omega}(\cdot, 0)\right)$ is the Minkowski functional of $\Omega$.

6. Symmetry and pluricomplex geodesics. Although the pluricomplex Green function displays a number of similarities to the classical Green function, there are significant differences. Perhaps the most important difference is the fact that pluricomplex Green functions are usually lacking symmetry (see Example 2.3). The first example of a non-symmetric pluricomplex Green function was published in $[\mathrm{BD}]$. It can be described as follows.

EXAMPle 6.1. Consider the function

$$
u(z, w)=\max \left\{\frac{1}{2} \log \frac{\left|w^{2}-z^{2}(z-c)\right|}{\varepsilon^{2}}, \log |z|\right\}, \quad(z, w) \in \mathbb{C}^{2},
$$

where $c \in \mathbb{D} \backslash\{0\}$ and $\varepsilon>0$ are fixed. Let $\Omega=\{u<0\}$. It is not difficult to check that $g_{\Omega}(z, 0)=u(z)$ for $z \in \Omega$. For instance, one can use the formula obtained in Example 5.3 with $d=1, n=2, a_{1}=1, a_{2}=1 / \varepsilon^{2}$ and $f_{11}(z, w)=-(z-c) / \varepsilon^{2}$. Then it can be shown that $g_{\Omega}((c, 0),(0,0))<g_{\Omega}((0,0),(c, 0))$, provided that $\varepsilon$ is sufficiently small (see $[\mathrm{BD}]$ or $[\mathrm{K} 3])$.

In direct analogy to complex geodesics used in the theory of invariant pseudodistances (see e.g. [D, DT, JP2, V1, V2, V3]) one can introduce the notion of pluricomplex geodesics related to pluricomplex Green functions. Let $\Omega$ be a bounded domain in $\mathbb{C}^{n}$. A holomorphic mapping $\varphi: \mathbb{D} \longrightarrow \Omega$ is called a pluricomplex geodesic if

$$
g_{\Omega}(\varphi(\zeta), \varphi(\xi))=g_{\mathbf{D}}(\zeta, \xi)
$$

for all $\zeta, \xi \in \mathbb{D}$. If $p, q \in \varphi(\mathbb{D})$, and $p \neq q$, we say that $p$ and $q$ can be joined by $a$ pluricomplex geodesic in $\Omega$. Notice that the existence of pluricomplex geodesics is connected with the problem of symmetry of the pluricomplex Green functions. 
Namely, if $\varphi$ is a pluricomplex geodesic, then the restriction of $g_{\Omega}$ to the set $\varphi(\mathbb{D}) \times \varphi(\mathbb{D})$ is symmetric. This implies that if for some $\Omega, p, q$ we have $g_{\Omega}(p, q) \neq$ $g_{\Omega}(q, p)$, then there is no pluricomplex geodesic in $\Omega$ joining $p$ and $q$.

Let $C_{\Omega}$ denote the Carathéodory pseudodistance in $\Omega$. If $\Omega$ is convex, then according to the well-known result of Lempert (see [L2] or [JP2]) the Carathéodory and Kobayashi distances coincide, and hence we have $\log \tanh C_{\Omega}=g_{\Omega}$ (see Theorem $2.3(\mathrm{x})$ ). Consequently in this case the notions of complex and pluricomplex geodesics coincide.

The definition of pluricomplex geodesics yields a number of corollaries.

Corollary 6.3. If $\varphi$ is a pluricomplex geodesic in $\Omega$, then for each $p \in \varphi(\mathbb{D})$ the function $\left\{\zeta \mapsto g_{\Omega}(\varphi(\zeta), p)\right\}$ is harmonic in $\mathbb{D}$.

COROLlaRY 6.4. Every pluricomplex geodesic is a proper holomorphic embedding. In particular, the range of a pluricomplex geodesic in $\Omega$ is a closed onedimensional submanifold of $\Omega$.

In view of Proposition 6.11 in [D] and Corollary 6.5.3 in [K3], we also have the following property.

Corollary 6.5. Let $\Omega$ be a domain in $\mathbb{C}^{n}$. Every complex $C_{\Omega}$-geodesic is a pluricomplex geodesic in $\Omega$.

Proposition 6.6. Let $\Omega$ be a bounded domain in $\mathbb{C}^{n}$, and let $\varphi: \mathbb{D} \longrightarrow \Omega$ be a holomorphic mapping such that there exist $\zeta, \xi \in \mathbb{D}$ for which

$$
g_{\Omega}(\varphi(\zeta), \varphi(\xi))=g_{\mathbf{D}}(\zeta, \xi) .
$$

If the function $\left\{\lambda \mapsto g_{\Omega}(\varphi(\zeta), \varphi(\lambda))\right\}$ is subharmonic in $\mathbb{D}$, then $\varphi$ is a pluricomplex geodesic.

Proof. Define

$$
u(\lambda)=g_{\Omega}(\varphi(\lambda), \varphi(\xi))-g_{\mathbb{D}}(\lambda, \xi), \quad \lambda \in \mathbb{D} \backslash\{\xi\} .
$$

Clearly, $u$ is subharmonic in $\mathbb{D} \backslash \xi$ and $u \leq 0$ (by Theorem 2.3(vi)). Hence $u$ extends to a non-negative subharmonic function in $\mathbb{D}$. Since $u(\zeta)=0$, the maximum principle implies that $u \equiv 0$. In other words

$$
g_{\Omega}(\varphi(\lambda), \varphi(\xi))=g_{\mathbb{D}}(\lambda, \xi)
$$

for all $\lambda \in \mathbb{D}$. Now, in order to finish the proof, one can fix $\lambda$ and repeat the above argument using the second variable of the pluricomplex Green function.

Since $(z, w) \mapsto g_{\Omega}(z, w)$ is symmetric if and only if it is separately plurisubharmonic, we have the following property.

Corollary 6.7. Let $\Omega$ be a bounded domain in $\mathbb{C}^{n}$, and let $\varphi: \mathbb{D} \longrightarrow \Omega$ be a holomorphic mapping such that there exist $\zeta, \xi \in \mathbb{D}$ for which

$$
g_{\Omega}(\varphi(\zeta), \varphi(\xi))=g_{\mathbf{D}}(\zeta, \xi) .
$$

If the function $g_{\Omega}$ is symmetric, then $\varphi$ is a pluricomplex geodesic. 
Proposition 6.8. Let $\Omega$ be a bounded domain in $\mathbb{C}^{n}$, and let $\varphi: \mathbb{D} \longrightarrow \Omega$ be a holomorphic mapping such that there exist $\zeta, \xi \in \mathbb{D}$ for which

$$
g_{\Omega}(\varphi(\zeta), \varphi(\xi))=g_{f D}(\zeta, \xi) .
$$

If there exists a non-constant holomorphic function $h: \Omega \longrightarrow \mathbb{C}$ such that

$$
\left.g_{h(\Omega)}(h(\varphi(\zeta)), h(\varphi(\xi)))=g_{\Omega}(\varphi(\zeta)), \varphi(\xi)\right),
$$

then $\varphi$ is a pluricomplex geodesic.

Proof. The previous proposition implies that $h \circ \varphi$ is a pluricomplex geodesic in $h(\Omega) \subset \mathbb{C}$, which in this case means that $h \circ \varphi$ is a biholomorphic mapping from $\mathbb{D}$ onto $h(\Omega)$. Consequently, we have:

$$
g_{\mathbb{D}}\left(\lambda_{1}, \lambda_{2}\right)=g_{h(\Omega)}\left(h\left(\varphi\left(\lambda_{1}\right)\right), h\left(\varphi\left(\lambda_{2}\right)\right)\right) \leq g_{\Omega}\left(\varphi\left(\lambda_{1}\right), \varphi\left(\lambda_{2}\right)\right) \leq g_{\mathbb{D}}\left(\lambda_{1}, \lambda_{2}\right)
$$

for all $\lambda_{1}, \lambda_{2} \in \mathbb{D}$.

The extent to which the notion of pluricomplex geodesics is useful is yet to be assessed. At present it appears that even in the simplest case of convex domains complex geodesics (of any kind) are rather hard to describe explicitly (see e.g. $[\mathrm{DT}]$ or $[\mathrm{JP} 2])$.

\section{References}

[AP] M. Abate and G. Patrizio, Uniqueness of complex geodesics and characterization of circular domains, Manuscripta Math. 74 (1992), 277-297.

[A] A. Aytuna, On Stein manifolds $M$ for which $\mathcal{O}(M)$ is isomorphic to $\mathcal{O}\left(\Delta^{n}\right)$, Manuscripta Math., 62 (1988), 297-315.

[A1] K. Azukawa, Two intrinsic pseudo-metrics with pseudoconvex indicatrices and starlike circular domains, J. Math. Soc. Japan 38 (1986), 627-647.

[A2] —, The invariant pseudo-metric related to negative plurisubharmonic functions, Kodai Math. J. 10 (1987), 83-92.

[BL] T. Bagby and N. Levenberg, Bernstein theorems for harmonic functions, preprint, Indiana University, Bloomington, 1992.

[B] E. Bedford, Survey of pluri-potential theory, preprint, 1990.

[BD] E. Bedford and J.-P. Demailly, Two counterexamples concerning the pluri-complex Green function in $\mathbf{C}^{n}$, Indiana Univ. Math. J. 37 (1988), 865-867.

[BT1] E. Bedford and B. A. Taylor, The Dirichlet problem for a complex Monge-Ampère equation, Invent. Math. 37 (1976), 1-44.

[BT2] - - - A new capacity for plurisubharmonic functions, Acta Math. 149 (1982), 1-40.

[BT3] - - - Plurisubharmonic functions with logarithmic singularities, Ann. Inst. Fourier (Grenoble) 38 (1988), 133-171.

[BT4] - - - Uniqueness for the complex Monge-Ampère equation for functions of logarithmic growth, Indiana Univ. Math. J. 38 (1989), 455-469.

[BŁ] Z. Błocki, Estimates for the complex Monge-Ampère operator, preprint, Jagiellonian University, Cracow, 1992

[BR] F. T. Brawn, The Green and Poisson kernels for the strip $\left.\mathbb{R}^{n} \times\right] 0,1[$, J. London Math. Soc. 2 (1970), 439-454.

[C1] U. Cegrell, Capacities in Complex Analysis, Aspects of Mathematics, Vieweg, Wiesbaden, 1988. 
[C2] U. Cegrell, The symmetric pluricomplex Green function, this volume, 135-141.

[CLN] S. S. Chern, H. Levine and L. Nirenberg, Intrinsic norms on a complex manifold, in: Global analysis, papers in honour of K. Kodaira, University of Tokyo Press, 1969, 119-139.

[D1] J. -P. Demailly, Mesures de Monge-Ampère et caractérisation géométrique des variétés algébriques affines, Mém. Soc. Math. France 19 (1985), 1-125.

[D2] —, Mesures de Monge-Ampère et mesures pluri-sousharmoniques, Math. Z. 194 (1987), $519-564$.

[D] S. Dineen, The Schwarz Lemma, Oxford Math. Monographs, Clarendon Press, Oxford, 1989 .

[DG] S. Dineen and F. Gaughran, Maximal plurisubharmonic functions on domains in Banach spaces, preprint, University College Dublin, 1992.

[DT] S. Dineen and R. M. Timoney, Complex geodesics on complex domains, in: Progress in Functional Analysis, K. D. Bierstedt, J. Bonet, J. Horváth and M. Maestre (eds.), Elsevier, 1992, 333-365.

[H1] M. Hervé, Lindelöf's principle in infinite dimensions, in: Proceedings on Infinite Dimensional Holomorphy, T. L. Hayden and T. J. Suffridge (eds.), Lecture Notes in Math. 364, Springer, Berlin, 1974, 41-57.

[H2] - Analyticity in Infinite Dimensional Spaces, de Gruyter Stud. Math. 10, Walter de Gruyter, Berlin, 1989.

[JP1] M. Jarnicki and P. Pflug, Invariant pseudodistances and pseudometrics - completeness and product property, Ann. Polon. Math. 60 (1991), 169-189.

[JP2] - - , Invariant Distances and Metrics in Complex Analysis, Walter de Gruyter \& Co, 1993.

[K] C. O. Kiselman, Sur la définition de l'opérateur de Monge-Ampère complexe, in: Analyse complexe, proceedings, Toulouse 1983, E. Amar, R. Gay, and Nguyen Thanh Van (eds.), Lecture Notes in Math. 1094, Springer, Berlin, 1983, 139-150.

[K1] M. Klimek, Extremal plurisubharmonic functions and invariant pseudodistances, Bull. Soc. Math. France 113 (1985), 231-240.

[K2] —, Infinitesimal pseudometrics and the Schwarz lemma, Proc. Amer. Math. Soc. 105 (1989), 134-140.

[K3] —, Pluripotential Theory, London Math. Soc. Monographs (New Series), Clarendon Press, Oxford, 1991.

[KR] S. L. Krushkal, The Green function of Teichmüller spaces with applications, Bull. Amer. Math. Soc. 27 (1992), 143-147.

[L] P. Lelong, Fonction de Green pluricomplexe pour les espaces de Banach, J. Math. Pures Appl. 68 (1989), 319-347.

[L1] L. Lempert, La métrique de Kobayashi et la représentation des domaines sur la boule, Bull. Soc. Math. France 109 (1981), 427-474.

[L2] - Holomorphic retracts and intrinsic metrics in convex domains, Anal. Math. 8 (1982), 257-261.

[L3] - Solving the degenerate Monge-Ampère equation with one concentrated singularity, Math. Ann. 263 (1983), 515-532.

[M] S. Momm, Boundary behaviour of extremal plurisubharmonic functions, preprint, University of Düsseldorf, 1992.

[PS] E. A. Poletskiu and B. V. Shabat, Invariant metrics, in: Encyclopaedia of Mathematical Sciences 9, Several Complex Variables III, G. M. Khenkin (ed.), trans. J. Peetre, Springer, Berlin, 1989, 63-111.

[RT] J. Rauch and B. A. Taylor, The Dirichlet problem for the multidimensional MongeAmpère equation, Rocky Mountain J. Math. 7 (1977), 345-364. 
[S] A. Sadullaev, Plurisubharmonic measures and capacities on complex manifolds, Russian Math. Surveys 36 (4) (1981), 61-119.

[SE] S. Semmes, A generalization of Riemann mappings and geometric structures on a space of domains in $\mathbf{C}^{n}$, Mem. Amer. Math. Soc. 472 (1992).

[SI] N. Sibony, Quelques problèmes de prolongement de courants en analyse complexe, Duke Math. J. 52 (1985), 157-97.

[V1] E. Vesentini, Variations on a theme of Carathéodory, Ann. Scuola Norm. Sup. Pisa 4 (1979), 39-68.

[V2] -, Complex geodesics, Compositio Math. 44 (1981), 375-394.

[V3] - Complex geodesics and holomorphic maps, Symposia Math. 26 (1982), 211-230.

[Z] A. Zeriahi, Fonction de Green pluricomplexe à pôle à l'infini sur un espace de Stein parabolique et applications, Math. Scand. 69 (1991), 89-126. 